\title{
A New Approach based on Fuzzy MADM for Enhancing Infrastructure Selection in the Case of VANET Network
}

\author{
Abdeslam Houari $^{1}$, Tomader Mazri ${ }^{2}$ \\ Laboratory of Advanced Systems Engineering, Ibn Tofail Science University \\ Kenitra, 14020, Morocco
}

\begin{abstract}
The emergence of the 5G mobile network has a huge impact on the evolution of services and functionalities offered to its customers; this latest version of mobile networks will allow the simultaneous connection of a significant number of people and IoT devices, in addition to the improvement of several other features. 5G will serve in a large part of smart cities and especially in the field of intelligent transportation systems (ITS). Vehicular Ad-Hoc Network (VANET) is one of the promising projects on which the ITS is relying on. Its main purpose is to provide communication and information-sharing support for the vehicles in its network. VANET is based on a heterogeneous network architecture composed mainly of two infrastructures, the first one is the cellular infrastructure, and the second is the road infrastructure. This paper proposes a new approach based on Fuzzy multiple attribute decision making (MADM) methods for the selection of the most appropriate infrastructure in the VANET network and consequently enhance the number of executed vertical handover to move from one infrastructure to another without loss of connection.
\end{abstract}

Keywords-5G; Vehicular adhoc network; internet of things; intelligent transportation system; cellular infrastructure; road infrastructure; fuzzy; vertical handover; MADM

\section{INTRODUCTION}

Nowadays, improving the vehicle's autonomy level has become one of the major concerns of the automotive sector. This new orientation is dictated by the ever-increasing number of accidents and traffic jams, which have irreversible social, ecological, and economic consequences. For this reason, the automotive sector is gradually turning to intelligent transportation systems (ITS).

VANET (vehicular ad-hoc network) is an important project related to ITS, a specific case of MANET (Mobile Ad-hoc Networks) network in which nodes are smart vehicles. The idea behind it is to connect the vehicles and share data and resources between them to understand their surroundings better and deal with the unexpected events that may occur on the road, such as traffic jams, which are one of the biggest issues for road users. Two main communication modes are provided; vehicles can communicate with each other via the V2V (vehicle-to-vehicle) mode using devices such as AU (application unit) or OBUs (On-Board Units), as they can communicate with the road infrastructure using the V2I (vehicle-to-infrastructure) mode which allows them to communicate with RSUs and Base station installed along the road to benefit from several services such as internet access. The high node mobility is one of the main characteristics of VANET networks caused by the random movement and high speed of the vehicles, which directly impacts the response time and the topology of the network.

The emergence of $5 \mathrm{G}$ mobile networks is highly expected by smart cities and especially intelligent transportation. This new generation of mobile networks is equipped with a set of advanced features, such as higher capacity in comparison to 4G, allowing device-to-device communication and providing a better density of mobile broadband users [1]. As a result, vehicles moving in such a heterogeneous network will benefit from various services offered by both infrastructures. Therefore, the network's right choice is an important step to improve the quality of services (QoS) and the vehicles' efficiency. The heterogeneous nature of the VANET network raises the challenge of service continuity; as a result, the performance of this type of network requires the effective use of a vertical handover process to know the most appropriate infrastructure according to predefined criteria. Generally, the selection process can be composed of three phases: data collection, decision transfer, and transfer execution after conducting a comparative study in the previous work presented at the ISAECT conference [2]. In this paper, we focus on the decision-making step. The decision-making in the VHO process depends on several QoS network parameters and criteria such as network state, capacity, reliability, and others. In order to solve the problem of the multitude of criteria that increases with each network upgrade, the multiple attribute decision-making (MADM) approach is proposed in this study. MADM is considered a promising solution to the network and infrastructure selection problem. It is simple to implement and does not require any particular physical resources. The bestknown algorithms of the MADM are the hierarchical analysis algorithms such as AHP (analytic hierarchy process) or the analytic network process (ANP) used in the criteria evaluation and weighting step, as well as VIKOR Saw and TOPSIS for ranking the available choices (networks or infrastructures). In the first part of this article, a presentation of the VANET network is performed, as well as its different characteristics, the modes of communication, the main components as well as the main challenges it faces. The next section describes the cellular infrastructure in the 5G era while detailing the main technologies that characterize it compared to previous generations of mobile networks. The following section is 
dedicated to the proposed approach, which explains the implemented system model and the applied methods for ranking the two infrastructures. The penultimate part concerns the simulations performed as well as the discussion of the results obtained. Finally, the article ends with a conclusion.

\section{VANET NETWORK}

As explained earlier, VANET is a form of MANET network, enabling data exchange communication between vehicles in a specific geographical area and between vehicles and equipment installed at the roadside, known as roadside equipment. Several industries are interested in this project, especially the transport sector, mainly for the safety aspect that it promises to ensure by reducing the number of accidents and traffic jams, positively impacting the ecology by reducing harmful gas emissions from road traffic. In this section, a focus on the different modes of communication supported by the VANET network is highlighted. After that, the main equipment on which it is based are listed; later, we explain the data processing cycle. We end with the main features and challenges it faces.

\section{A. Communications Mode}

In a VANET network (Fig. 1), vehicles communicate with each other through the vehicle-to-vehicle mode and with road equipment through the V2I (vehicle-to-infrastructure) mode; this will allow drivers and authorities to benefit instantaneously from different information on road conditions and to take advantage of several other services offered by the network (entertainment applications, games, localization...) allowing drivers and passengers to travel safely and comfortably. There is also another type of communication, which not only interconnects vehicles and infrastructure but also includes cellular networks and other devices, this new mode called Vehicle-to-everything (V2X) is composed of V2V, V2I modes in addition to V2P Vehicle-to-Pedestrian (V2P), and Vehicleto-Device (V2D).

Furthermore, VANET networks are essentially based on two types of applications, one constituting the core of the ITS, which ensures the improvement of road safety, and the second type of application for the comfort of the driver and his passengers[3].

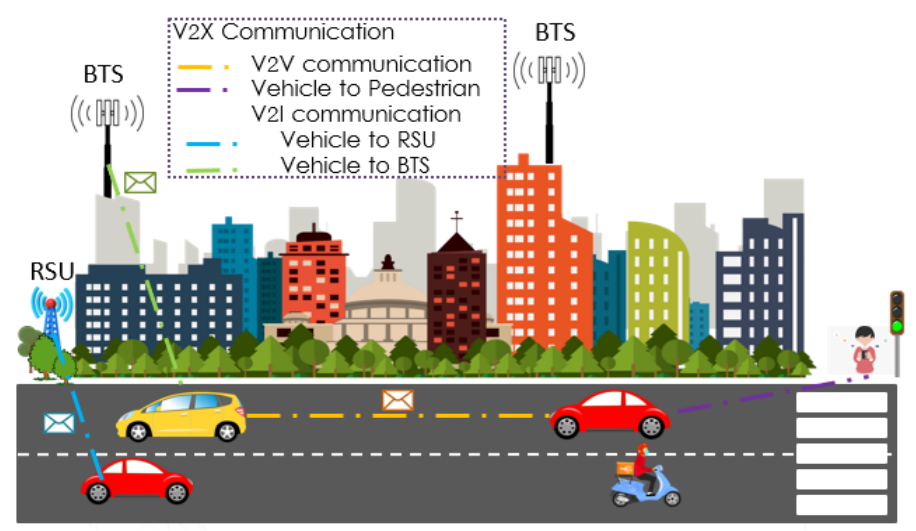

Fig. 1. Communication Modes.

\section{B. Network Architecture Component}

The data exchange at the network layer is handled by DSRC/WAVE wireless medium, whose role is to secure data transfer and reduce latency. The resulting communication leads to more reliable information and allows the deployment of safety applications that enhance travelers' safety and make driving more pleasant. Among the main components of the VANET network, there are:

- OBU: Commonly used with the RSU or other OBUs, this device is placed on the vehicle's board. Its role is to share information with the other vehicles. It is also used to retrieve information from the sensors installed in the vehicle. The connection with the RSU and other OBUs is established through an IEEE 802.11p wireless $\operatorname{link}[4]$.

- AU: The Application unit exploits the communication means provided by the OBU in order to communicate with the network[4]. It is used in various applications; it can be used for security applications and internet access devices such as PDA (personal digital assistants).

- RSU: the road side-unit is a wave device used to extend the range of communication. It is also used to send warning messages on the road to prevent accidents[4].

- Base Station: It is equipment whose role is to connect mobile devices to access a telecommunication network; it comprises a transmission antenna and a reception antenna.

The VANET network vehicles are equipped with a set of sensors enabling them to discover their surroundings better in order to make the right decisions at the right moment. To do this, the vehicle starts by collecting data from its surroundings (location, road conditions, accident, etc.) via the sensors and cameras installed onboard. These data are then stored and processed to retain only the most important ones. Finally, the process ends by transmitting this data to the recipient vehicle belonging to the same network or a completely different network type (Fig. 2).

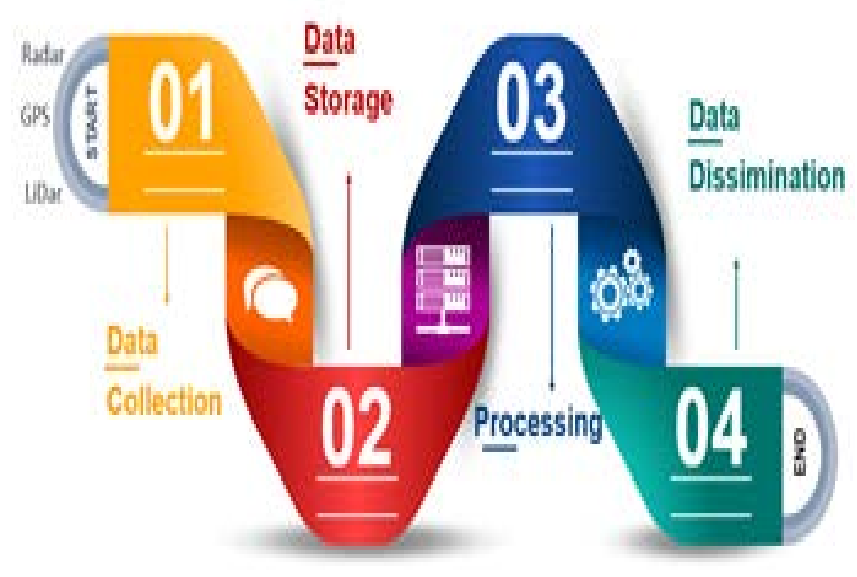

Fig. 2. Data Collection Process. 


\section{VANET Features}

As mentioned above, VANET network is a specific case of a MANET network with features that differentiate it from other mobile networks, such as:

- Dynamic topology: this specificity comes from the fact that the vehicles are constantly changing their position and driving at high speed. They can also move in both directions and join or leave the network at any moment.

- Frequent disconnection: this is one of the direct consequences of the network's dynamic topology, making the connection unstable. The link between vehicles can easily be broken, resulting in the loss of the data exchanged.

- Mobility and prediction: Although the network's topology is dynamic, vehicles normally follow a mobility model that considers traffic lights, speed limits, road conditions, and driver behavior. However, the vehicle trajectory cannot be predicted in advance[5].

- Geographical communication: communication between a source and destination vehicles is possible only if the latter can be reached, a method quite different from those used in other types of networks, which are based more on the vehicle Id.

These different features have led to new challenges in setting up a safe and reliable exchange system that takes into account the heterogeneous aspect of the network and its infrastructure, without forgetting the particularity of the vehicles considered as important storage resources and powerful processing units.

\section{Challenges in VANET Network}

VANET faces a wide range of challenges (Fig. 3). The most critical ones are data privacy and security, especially as messages are broadcast. Anyone can intercept them, making the network vulnerable to various attacks that threaten road users' safety [6]. Security is an important aspect of any system. Therefore, it must fulfill certain requirements for the system's proper functioning and the continuity of its services. Failure to comply with any of these requirements may lead to irreversible consequences, such as a system crash or data loss.

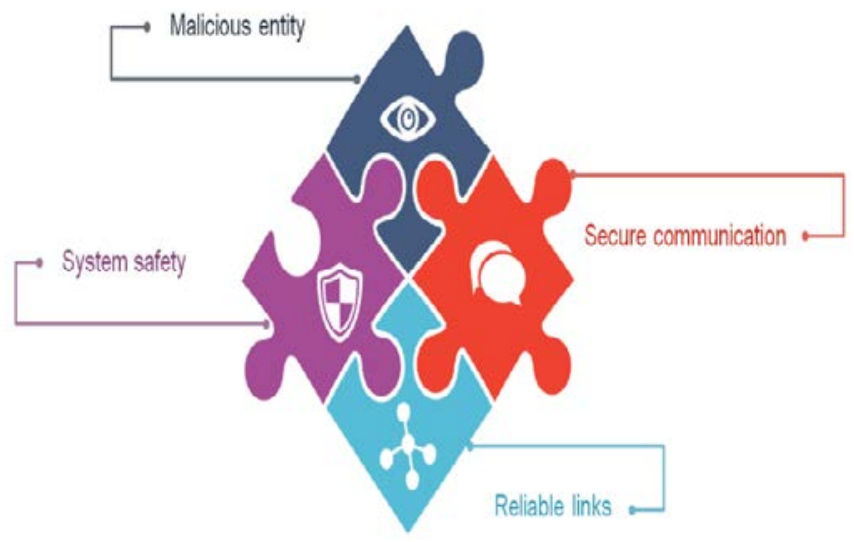

Fig. 3. VANET Challenges.
Moreover, other requirements are exclusive to VANET networks, such as authentication, data integrity, and confidentiality. Authentication in VANET networks aims to monitor the vehicle's authentication levels. Once connected, the vehicle has a unique identifier, which prevents it from passing for multiple vehicles and usurping other vehicles' identities [7]. The second one is integrity; its role is to ensure that the message's data has not been altered or modified fraudulently during its transmission to the receiving node. For confidentiality, its role is to ensure the reliability of the communication. Sending encrypted messages ensures that only the destination vehicle is authorized to consult their content[7]. The security criterion aims to secure communications between vehicles on the one hand and between vehicles and road facilities on the other hand. One of the most dangerous attacks that vehicles face is the Sybil Attack, which is the subject of several research and studies. one of the recommended ways to prevent this attack is to establish a balance between privacy and non-repudiation[8]. Other dangerous attacks can target vehicles, such as jamming and DOS attacks, which require further research to discover the appropriate security mechanisms and algorithms to protect against them.

In some cases, the communication in an open space leads to the infiltration of malicious nodes that can intercept and corrupt the network's data. One of the major challenges that VANET faces is preventing attackers from accessing information to preserve the reliability of the links set up between vehicles and the loss of data packets on the network. The author in [9] have implemented the Reputation-Based Global Trust Establishment (RGTE) scheme to achieve these objectives. The method adopted is based on statistical laws to circulate only trustworthy information. It also allows the detection of malicious nodes through a dynamic mechanism. However, this method cannot be applied in high mobility vehicles due to packet loss.

Finally, the last major challenge for VANET networks is the link reliability between vehicles in V2V and V2I communications used for traffic data collection. Due to the high node mobility, this problem persists until now. One of the proposals is to deploy a system based on IDS (Intrusion Detection Scheme) for detecting malicious nodes involved in illegal activities. In this scheme, each node is linked to a control system in order to monitor each node and check if the network packet is transferred to the next node. Unfortunately, there is a strong probability that the packets are lost and do not reach their destination, and these nodes are consequently considered malicious even if they are not in reality. Note that this type of system has not yet been implemented and is just at the experimentation phase.

\section{CELLULAR INFRASTRUCTURE IN THE 5G ERA}

Cellular networks appeared at the beginning of the 1990s, its objective is to provide voice service, using a set of base stations installed in the coverage area, each of these stations cover a smaller area exclusively. The addition of traffic and data processing have been introduced into mobile networks from the second generation onwards. Mobile networks have undergone several evolutions, not just in terms of bandwidth but also in terms of standards and technologies. Several 
components constitute this network. Among them, there are two that have remained the same throughout the years. The first is the Radio Access Network (RAN), and the second is the Core Network (CN). The RAN is responsible for wireless signal processing (baseband, passband) from the user equipment (UE). Simultaneously, the $\mathrm{CN}$ is responsible for the incoming and outgoing traffic flow's reliable routing to their destinations.

\section{A. Main Components of the Network}

During the development of mobile networks, several components have evolved many times. The eNodeB (the evolved node B) represents the base station covering the user equipment in a defined area, through which the user equipment can communicate and reach the remote destination. The other component is the Tracking Area (TA), which manages mobility and optimizes user and system management. In practice the area covered is partitioned into several TAs, and each of them includes a set of enodeBs. The Mobility Management Entity (MME) is one of the most important control components that are part of the LTE network; it acts as a signalling node in an EPC control plane.Finally, the Serving Gateway (SGW) component acts as a router and allows the Transfer of the data flow between the BSs and the Packet Data Network Gateway. A typical architecture of a cellular network can be illustrated as follows (Fig. 4).

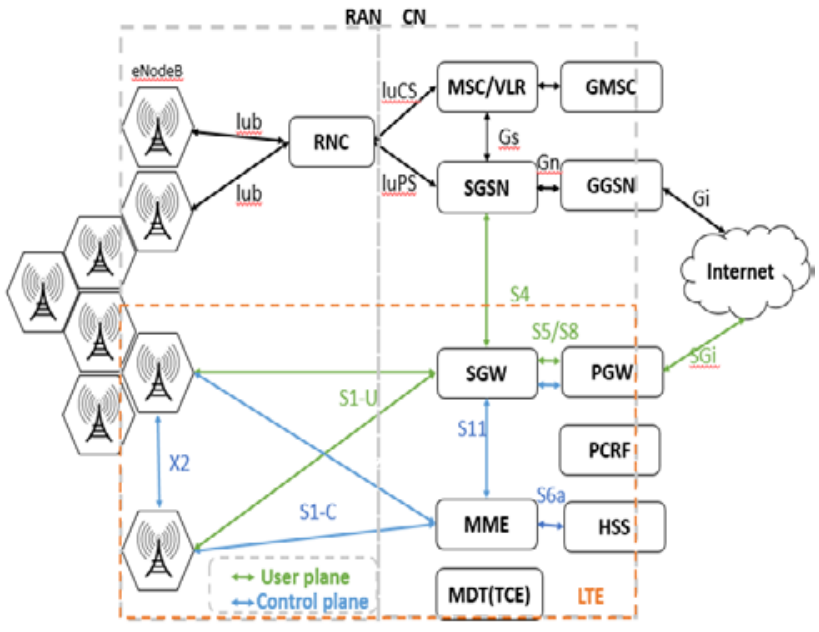

Fig. 4. Cellular Network Architecture.

\section{B. Overview of the $5 G$}

In the case of VANET networks, the data exchange occurs several times and between different actors. The generated data requires special processing, as it contains valuable information on road traffic. Unfortunately, conventional data management systems do not allow the management of a large amount of data in a short period. This new generation of mobile networks promises to solve several problems, including massive data management. Indeed, 5G has started to invade the global market since 2019; several new features will emerge, such as improved response time (estimated at 1ms). 5G will also enable the massive deployment of the Internet of Things (IoT) ecosystem in where networks will satisfy the communication needs of billions of connected devices in a very short time and at a low cost while reducing energy consumption. Several technologies constitute the pillars of 5G (Fig. 5), enabling it to achieve a level of performance unequaled in the history of mobile networks; the first is the small cell, which is a cellular radio access node with a range from 10 meters to a few kilometers, its role is to increase the data capacity of the network, since, as it is the case with the previous generations, $5 \mathrm{G}$ radio waves cannot reach a long-range due to the high frequency used by 5G. The mmWaves (millimeter wave) are localized between $30 \mathrm{GHz}$ and $300 \mathrm{GHs}$; using these waves allows the $5 \mathrm{G}$ to reach a high transmission speed. For BeamForming, it is used to steer a radio wave to a specific destination; this is done by tuning the radio waves in order to point in a particular direction. The penultimate technology, which is the Massive mimo, aims to transmit $\mathrm{M}$ data flows simultaneously on $\mathrm{M}$ transmitting antennas, and each flow is received by $\mathrm{N}$ receiving antennas; for this purpose, often several transmitting and receiving antennas are used to improve both the transmission gain and the spectral efficiency. Finally, full duplex is a technology that allows a transceiver to transmit and receive data at the same time, this last technology enables to increase the capacity of the wireless network at the physical layer.

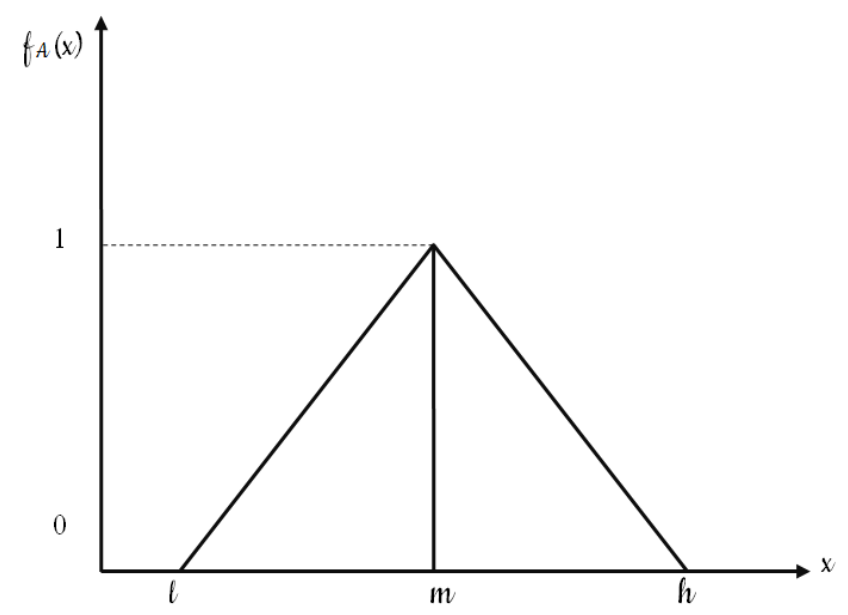

Fig. 5. Triangular Fuzzy Number.

\section{Proposed Approach to Select the Best Network}

Roaming between the two infrastructures, road, and cellular is essential for any vehicle belonging to the VANET network qualified as a heterogeneous network. The selection between the two infrastructures is quite an important step. Unlike traditional mobile networks, the nodes' mobility directly impacts the network topology and affects the performance of the used protocols [10]. Also, in urban areas with high towers and a growing number of buildings, the V2X connections can be disturbed due to the phenomenon of channel fading, which is spatially correlated with the number of fixed obstacles locations [11]. In addition, the mobility of vehicles has an impact on the stability of the cluster node due to its effect on the merge and the split of the cluster [12]. As a result, vehicle mobility faces technical and organizational challenges in maintaining connections between vehicles in V2V mode on the one hand and between vehicles and infrastructure (RSU/BS) on the other hand. Moreover, since the vehicle constantly changes 
position and moves in and out of the coverages of other vehicles, RSUs, and BSs.

The management of mobility becomes essential to maintain the continuity of shared services and to achieve better QoS in a heterogeneous environment. Hence the importance of using handover strategies and an appropriate network selection scheme. There are two types of handover strategy, the first is horizontal and concerns the data session transmission from one PoA to another in the same network, the second strategy is the vertical handover [13], used when a data transmission session is transmitted from one PoA to another belonging to another network (using a completely different access technology).

\section{A. Choice of Criteria}

The selected criteria have been retained since they cover the different aspects that a network should satisfy.

Capacity must be significant to meet the increased demand for bandwidth. In fact, transmitting alerts and information messages requires a moderate capacity since they are transferred occasionally. Nevertheless, autonomous driving systems require a regular exchange with vehicle onboard sensors, in addition to the applications used by road users, which consume a considerable amount of data. For the second criteria, VANET network security faces several challenges, especially with IoT integration, which will increase the number of end-users and connected objects. Different types of attacks have emerged and evolved over time, such as availability and data integrity attacks, which have irreversible consequences on vehicles and people's data and can sometimes lead to road users' death, hence the importance of taking this criterion into account in this study. On the other hand, Coverage is a determining criterion in the infrastructure selection because vehicles will be able to communicate with the infrastructure only if they are within the coverage area of the BS and RSU. Studies have shown that $4 \mathrm{G}$ coverage is better than $5 \mathrm{G}$ coverage, especially as the latter uses small cell technology. For this reason, during the simulation, the RSU coverage was given an advantage over the 5G coverage. Finally, for the last two criteria, in the VANET context, several constraints slow down the response time (mainly high mobility and dynamic network topology) and others that affect the reliability of the data exchanged, and what has been noticed in the tests is that improving reliability harms performance and latency and vice versa.

\section{B. MADM Methods}

In the context of the heterogeneous environment, there are several types of MADM methods (SAW, GRA, TOPSIS, AHP, $\mathrm{MEW}$ ) that have proved their efficiency in solving VHO decisions mainly for the simplicity of their implementation. According to researchers, the most efficient methods used in the VHO process are TOPSIS, SAW, and VIKOR[14]. The principle on which they are based is ranking the candidate networks according to their scores. The researchers[15] proposed given weights in order to know the importance of each attribute in the QoS class under consideration. For other researchers, such as [16],they studied using AHP/TOPSIS pair for decision making situation, AHP to calculate the vector weight and TOPSIS for ranking access network, and their results showed the importance of the weights in decision making. Concerning decision-making, algorithms based on intelligent computation are the most performant, using a neural network and intelligent implementation techniques such as fuzzy logic. Indeed,[17] have been interested in the Fuzzy AHP to determine the weight of the evaluation criteria and the Fuzzy TOPSIS for classifying the alternatives. For these reasons, we have included fuzzy logic in the model to ensure the appropriate network selection.

\section{System Model}

This study focuses on the proposal of a MADM approach to evaluate the two alternatives subject to this paper: cellular and road infrastructure. To do so, we start by identifying the alternatives and the evaluation criteria, and then we build the hierarchy of decisions. The criteria retained to carry out the handover decision are those of capacity, security, coverage, reliability, and latency. Finally, TOPSIS is applied to the weighted matrices to obtain the ranking of the two infrastructures. One thousand iterations covering the different weight vectors have been performed (Fig. 6).

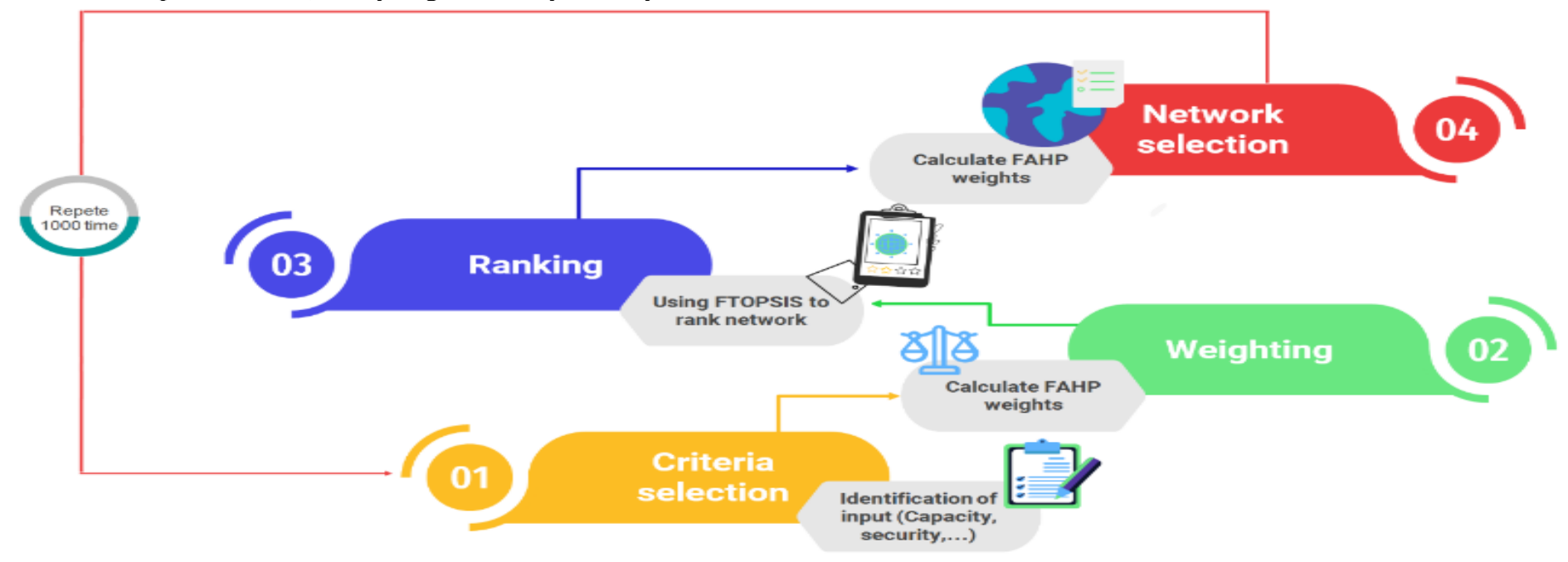

Fig. 6. System Model. 


\section{Network Selection using Fuzzy MADM Approach}

To deal with the uncertainty of human decisions and thoughts, Zadeh [18] introduced the fuzzy set theory to represent the vagueness relative to certain classes of objects mathematically. Its ability to represent vague data is considered an important contribution in the field of mathematics and technology in general.

A fuzzy set (A in $\mathrm{X}$ ) is characterized by a membership function $f$ which associates for each point belonging to $\mathrm{X}$ a real number in the interval [0.1], $f_{A}(\mathrm{x})$ represents the membership grade of $\mathrm{x}$ in $\mathrm{A}$.

$f_{A(x)}= \begin{cases}\frac{x-l}{m-l}, & l<x<m \\ \frac{h-x}{h-m}, & m<x<h \\ 0 \quad, & \text { Otherwise }\end{cases}$

In this case, TFN (Triangular fuzzy number) will be used in order to present fuzzy relative importance (Fig 7). In the pairwise comparison the TFNs used are defined by three real numbers expressed as a triplet $(1, m, h)$ with $l \leq m \leq h$ in order to describe a fuzzy event. The choice of TFN is generally related to the number of tuning and classifications (Table 1).

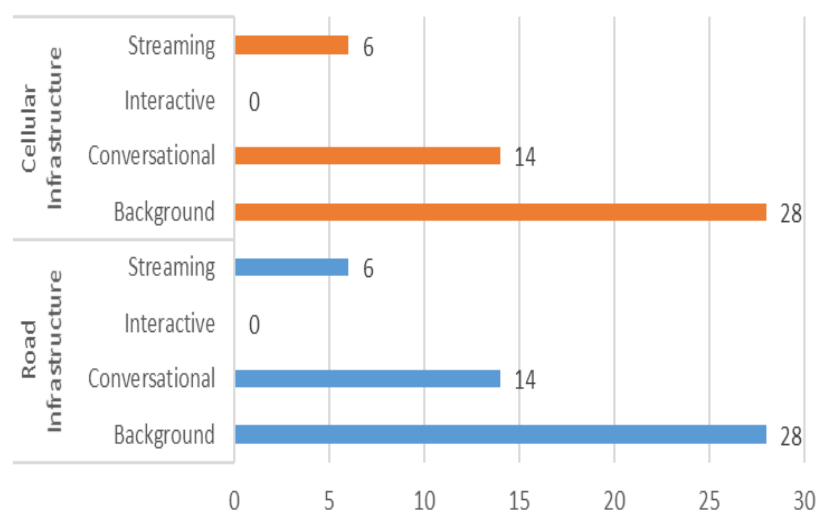

Fig. 7. Number of Vertical Handovers for 1000 Iterations.

TABLE I. MEMBERSHIP FUNCTION OF LINGUISTIC SCALE

\begin{tabular}{|l|l|l|}
\hline $\begin{array}{l}\text { Fuzzy } \\
\text { Number }\end{array}$ & Linguistic scales & TFN \\
\hline$\tilde{1}$ & Equally important (Eq) & $(1,1,1)$ \\
\hline$\tilde{3}$ & Weakly important (Wk) & $(2,3,4)$ \\
\hline$\tilde{5}$ & Essentially important (Es) & $(4,5,6)$ \\
\hline$\tilde{7}$ & Very strongly important (Vs) & $(6,7,8)$ \\
\hline$\tilde{9}$ & Absolutely important (Ab) & $(9,9,9$ \\
\hline
\end{tabular}

a) AHP: The AHP technique has been introduced to analyze and organize complex decisions. However, research has shown some weaknesses in the AHP Saaty method [19]. FAHP appeared to overcome this deficiency; this new method combines AHP using fuzzy logic and linguistic variables. The most important step in the FAHP process is the generation of the relative fuzzy importance for each pair of factors. Using TFN and through the pair-wise comparison, the following fuzzy valuation matrix is obtained:
$\tilde{V}=\left(\tilde{v}_{i j}\right)_{n * m}=\left(\begin{array}{ccc}(1,1,1) & \cdots & \tilde{v}_{1 m} \\ \vdots & \ddots & \vdots \\ \tilde{v}_{n 1} & \cdots & (1,1,1)\end{array}\right)$

Such as:

$\tilde{v}_{i j}=\left(l_{i j}, m_{i j}, u_{i j}\right)$

Concerning the process of weighing using the fuzzy AHP, we opted for the Bucklet's method [20]. The geometric mean method is used for the calculation and analysis of the resulting vector in the criteria of comparison. From the equation (1), the geometric mean procedure takes the following form:

$\widetilde{r}_{i}=\left(\tilde{v}_{i 1} \otimes \cdots \otimes \tilde{v}_{i m}\right)^{1 / m}$

Thereafter the calculation of the weights is given by:

$\widetilde{w}_{i}=\tilde{r}_{i} \otimes\left(\tilde{r}_{1} \oplus \cdots \oplus \tilde{r}_{m}\right)^{-1}$

The following equation is used to calculate the final resulting vector [21]:

$\widetilde{w}_{i}^{A / G}=\left(\widetilde{w}_{i}^{A / C_{1}} \otimes \widetilde{w}_{1}^{C / G}\right) \oplus \quad \cdots \oplus\left(\widetilde{w}_{i}^{A / C_{m}} \otimes \widetilde{w}_{m}^{C / G}\right)$

To complete, the fuzzy AHP method is applied to each of the QoS classes and weights are generated for each criterion.

b) TOPSIS: For a system selection requirement, a MADM method called TOPSIS (Technique for Order Preference by Similarity to Ideal Solution) was developed in 1981. It is a ranking technique that is simple to conceive and apply. TOPSIS aims to propose the best alternative that simultaneously has the shortest distance to the ideal positive solution and the farthest distance to the ideal negative solution[22]. the steps of TOPSIS have been detailed in [23]We make the normalized decision matrix of beneficial and non-beneficial criteria:

$$
p_{i j}=\frac{x_{i j}}{\sum_{i=1}^{n} x_{i j}}
$$

Concerning the weighted normalized decision matrix, it is calculated as follows:

$t_{i j}=W_{i} \cdot n_{i j}$ where $\sum_{j=1}^{n} W_{i}=1$

The ideal positive and negative solutions are:

$$
\begin{aligned}
& p^{+}=\left\{v_{1}^{+}, \ldots v_{2}^{+}, \ldots\right\}, v_{j}^{+}=\max _{i}\left(v_{i j}\right) \\
& p^{-}=\left\{v_{1}^{-}, \ldots v_{2}^{-}, \ldots\right\}, v_{j}^{-}=\min _{i}\left(v_{i j}\right)
\end{aligned}
$$

The distances that separate each Alternative $\mathrm{P}$ from the positive ideal solution and the separation of this alternative from the negative anti-ideal solution are calculated as follows:

$$
\begin{aligned}
& d_{i}^{+}=\sqrt{\sum_{j=1}^{M}\left|v_{i}^{+}-v_{i j}\right|} \\
& d_{i}^{-}=\sqrt{\sum_{j=1}^{M}\left|v_{i}^{-}-v_{i j}\right|}
\end{aligned}
$$

E. Finally, we Calculate the Relative Proximity to the Ideal Solution by Applying the Following Formula:

$0 \leq R_{i}=\frac{d_{i}^{+}}{d_{i}^{+}+d_{i}^{-}} \leq 1$ 
a) QoS Class: In this study, we have retained three main classes from the IEEE 802.16 Broadband Wireless Access Working Group project: conversational, streaming, interactive, and background class. The distinguishing factor between these classes is the delay sensitivity of the traffic. For example, for the conversational class, it is dedicated to traffic, which is very sensitive to delay, unlike the background class, which is much less delay-sensitive.

The different applications used on the road network are divided into these classes according to their delay sensitivity but also in terms of their need in terms of bandwidth and the level of security required, for this purpose we have ordered each criteria by level of importance for each class by assigning each one a TFN, thereafter we have calculated their weights which we will show in a table in the following section.

\section{EXPERIMENTAL RESULT}

\section{A. Methodology}

The simulations designed for the two infrastructure systems, Road and Cellular based on 5G, involved four application types (background, Streaming, Conversational, and Interactive) that cover different QoS requirements for the user side. For each of the available alternatives, the selected criteria were randomly generated according to the intervals mentioned in Table 3 for the thousand iterations of the experimentation. Finally, each range level was associated with a different vector weight. The criteria weights are calculated for each class of service using the fuzzy AHP method and are listed in Table 2.

TABLE II. ASSOCIATED WEIGHTS FOR EACH QOS CLASS

\begin{tabular}{|l|l|l|l|l|l|}
\hline QoS Class & $\begin{array}{l}\text { Capacit } \\
\mathbf{y}\end{array}$ & $\begin{array}{l}\text { Securit } \\
\mathbf{y}\end{array}$ & $\begin{array}{l}\text { Coverag } \\
\mathbf{e}\end{array}$ & $\begin{array}{l}\text { Reliabilit } \\
\mathbf{y}\end{array}$ & $\begin{array}{l}\text { Latenc } \\
\mathbf{y}\end{array}$ \\
\hline Background & 0,053 & 0,403 & 0,105 & 0,409 & 0,030 \\
\hline Streaming & 0,097 & 0,145 & 0,156 & 0,316 & 0,286 \\
\hline $\begin{array}{l}\text { Conversationa } \\
\text { l }\end{array}$ & 0,090 & 0,219 & 0,197 & 0,044 & 0,450 \\
\hline Interactive & 0,075 & 0,177 & 0,095 & 0,313 & 0,340 \\
\hline
\end{tabular}

TABLE III. COMPARISON CRITERIA

\begin{tabular}{|l|l|l|l|l|l|}
\hline Criteria & $\begin{array}{l}\text { Capacity } \\
\mathbf{( M b i t / s )}\end{array}$ & $\begin{array}{l}\text { Security } \\
\mathbf{( \% )}\end{array}$ & $\begin{array}{l}\text { Coverage } \\
\mathbf{( m )}\end{array}$ & $\begin{array}{l}\text { Reliability } \\
\mathbf{\%}\end{array}$ & $\begin{array}{l}\text { Latency } \\
(\mathbf{m s})\end{array}$ \\
\hline Road Infra. & {$[10,30]$} & 70 & {$[1,1000]$} & 40 & {$[10,100]$} \\
\hline $\begin{array}{l}\text { Cellular } \\
\text { Infra. }\end{array}$ & {$[1000,3000]$} & 50 & {$[1,100]$} & 50 & {$[1,10]$} \\
\hline
\end{tabular}

B. Results

The results of the first phase of tests performed on the two infrastructures for the different iterations of each scenario show that the approach adopted ensures an equal distribution of vertical handover decision between the two infrastructures for the different types of applications, except for interactive communications (Fig. 8). In fact, this proves that for $\mathrm{V} 2 \mathrm{~V}$ and V2I vehicle interactions, the mobile infrastructure is more adapted than the road network for this kind of exchange.

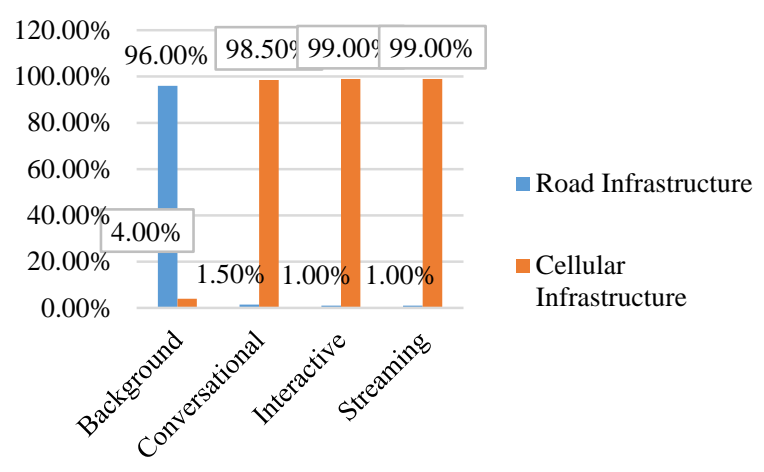

Fig. 8. The Average Number of Infrastructure Selections Per QoS Class.

For the second phase, the simulations showed that the cellular infrastructure exceeded the number of times it was selected, except in background applications, which revealed that vehicles tend to use the road infrastructure to run their programs in this particular case.

The received results demonstrate the potential benefit that $5 \mathrm{G}$ has over the other technologies used in VANET's V2X communications. This will have the effect of better managing the different types of communications as well as improving the services offered by each of the stakeholders in the VANET environment.

\section{CONCLUSION AND PERSPECTIVES}

This article proposes a new approach based on Fuzzy MADM for selecting the most appropriate infrastructure in a VANET network. The two infrastructures that are the subject of this study are the cellular and the road infrastructure. To conduct this comparative study, five criteria have been considered: capacity, security, coverage, reliability, and latency. We started by presenting the VANET network with its main components, characteristics, and different communication modes. We also introduced the 5G mobile infrastructure, highlighting the main technologies that distinguish it from the previous versions. Then we ended the article by the comparative study while explaining the system model adopted and the different methods used to test and evaluate the two infrastructures, namely the Fuzzy AHP, to generate the weights of the evaluation criteria, and the TOPSIS method was applied to rank the alternatives. The adopted approach also allows us to select better the infrastructure in a shorter delay and less complexity. This approach's advantage for managing vertical handover decision in this type of network characterized by its dynamic topology and its nodes' high mobility has also been demonstrated.

We intend to include vehicle mobility parameters during the tests to have a more realistic simulation in future work. We also plan to test other combinations of MADM methods such as VIKOR and SAW to select the best network and infrastructure.

\section{REFERENCES}

[1] A. Hussein, I. H. Elhajj, A. Chehab, et A. Kayssi, « SDN VANETs in 5G: An architecture for resilient security services », in 2017 Fourth International Conference on Software Defined Systems (SDS), Valencia, Spain, mai 2017, p. 67-74. doi: 10.1109/SDS.2017.7939143. 
[2] A. Houari et T. Mazri, « A comparative study of road infrastructure and 5G- based cellular infrastructure in the case of a VANET network. », p. 5.

[3] K. MOGHRAOUI, “ GESTION DE L’ANONYMAT DES COMMUNICATIONS DANS LES RÉSEAUX VÉHICULAIRES AD HOC SANS FIL (VANETs) ", Thesis, L’Université du Québec, Montréal, 2015. [En ligne]. Disponible sur: https://oraprdnt.uqtr.uquebec.ca/pls/public/docs/FWG/GSC/Publication/ 1645/34/1918/1/75448/8/F1689012680_M_moire_FINAL_K._Moghra oui.pdf.

[4] S. Al-Sultan, M. M. Al-Doori, A. H. Al-Bayatti, et H. Zedan, « A comprehensive survey on vehicular Ad Hoc network ", J. Netw. Comput. Appl., vol. 37, p. 380-392, janv. 2014.

[5] F. Cunha et al., " Data communication in VANETs: Protocols, applications and challenges ", Ad Hoc Netw., vol. 44, p. 90-103, juill. 2016.

[6] A. Houari, T. Mazri, et A. Addaim, « Using the static cloud vehicle for storing and caching data in VANET network ", in 2020 Fourth International Conference On Intelligent Computing in Data Sciences (ICDS), Fez, Morocco, oct. 2020, p. 1-7.

[7] M. N. Mejri, J. Ben-Othman, et M. Hamdi, « Survey on VANET security challenges and possible cryptographic solutions ", Veh. Commun., vol. 1, no 2, p. 53-66, avr. 2014.

[8] M. S. Sheikh, J. Liang, et W. Wang, « Security and Privacy in Vehicular Ad Hoc Network and Vehicle Cloud Computing: A Survey », Wirel. Commun. Mob. Comput., vol. 2020, p. 1-25, janv. 2020.

[9] X. Li, J. Liu, X. Li, et W. Sun, « RGTE: A Reputation-Based Global Trust Establishment in VANETs », in 2013 5th International Conference on Intelligent Networking and Collaborative Systems, Xi'an city, Shaanxi province, China, sept. 2013, p. 210-214.

[10] K. Abboud et W. Zhuang, Mobility Modeling for Vehicular Communication Networks. Cham: Springer International Publishing, 2015.

[11] E. Yaacoub, F. Filali, et A. Abu-Dayya, " QoE Enhancement of SVC Video Streaming Over Vehicular Networks Using Cooperative LTE/802.11p Communications », IEEE J. Sel. Top. Signal Process., vol. 9, no 1, Art. no 1, févr. 2015.
[12] K. Abboud et W. Zhuang, « Stochastic Modeling of Single-Hop Cluster Stability in Vehicular Ad Hoc Networks », IEEE Trans. Veh. Technol., vol. 65, no 1, Art. no 1, janv. 2016.

[13] S. Fernandes et A. Karmouch, " Vertical Mobility Management Architectures in Wireless Networks: A Comprehensive Survey and Future Directions », IEEE Commun. Surv. Tutor., vol. 14, no 1, Art. no 1, 2012.

[14] Z. Xu, Uncertain Multi-Attribute Decision Making. Berlin, Heidelberg: Springer Berlin Heidelberg, 2015. doi: 10.1007/978-3-662-45640-8.

[15] E. Stevens-Navarro et V. W. S. Wong, « Comparison between Vertical Handoff Decision Algorithms for Heterogeneous Wireless Networks », in 2006 IEEE 63rd Vehicular Technology Conference, Melbourne, Australia, 2006, vol. 2, p. 947-951.

[16] K. Savitha et C. Chandrasekar, « Trusted Network Selection using SAW and TOPSIS Algorithms for Heterogeneous Wireless Networks », Int. J. Comput. Appl., vol. 26, no 8, Art. no 8, juill. 2011.

[17] V. Sasirekha et M. Ilanzkumaran, « Heterogeneous wireless network selection using FAHP integrated with TOPSIS and VIKOR », in 2013 International Conference on Pattern Recognition, Informatics and Mobile Engineering, Salem, févr. 2013, p. 399-407.

[18] J. A. Goguen, « L. A. Zadeh. Fuzzy sets. Information and control, vol. 8 (1965), pp. 338-353. - L. A. Zadeh. Similarity relations and fuzzy orderings. Information sciences, vol. 3 (1971), pp. 177-200. », J. Symb. Log., vol. 38, no 4, Art. no 4, déc. 1973.

[19] R. W. Saaty, « The analytic hierarchy process - what it is and how it is used ", Math. Model., vol. 9, no 3-5, Art. no 3-5, 1987, doi: 10.1016/0270-0255(87)90473-8.

[20] J. J. Buckley, « Fuzzy hierarchical analysis », Fuzzy Sets Syst., vol. 17, no 3, Art. no 3, déc. 1985.

[21] A. Radionovs et O. Uzhga-Rebrov, « COMPARISON OF DIFFERENT FUZZY AHP METHODOLOGIES IN RISK ASSESSMENT », Environ. Technol. Resour. Proc. Int. Sci. Pract. Conf., vol. 2, p. 137, juin 2017.

[22] C.-L. Hwang et K. Yoon, Multiple Attribute Decision Making, vol. 186. Berlin, Heidelberg: Springer Berlin Heidelberg, 1981.

[23] A. Vega, J. Aguarón, J. García-Alcaraz, et J. M. Moreno-Jiménez, « Notes on Dependent Attributes in TOPSIS », Procedia Comput. Sci., vol. 31, p. 308-317, 2014. 